\title{
Adaptive synchronization of a class of fractional order chaotic system
}

\section{with uncertain parameters}

KONG De-fu, ZHAO Xiao-shan

(College of science, Tianjin University of Technology and Education, Tianjin P.R.C.)

\begin{abstract}
:
In this manuscript, the adaptive synchronization of a class of fractional order chaotic system with uncertain parameters is studied. Firstly, the local stability of the fractional order chaotic system is analyzed using fractional stability criterion. Then, based on the $\mathrm{J}$ function criterion, suitable adaptive synchronization controller and parameter identification rules of the unknown parameters are investigated. Finally, the numerical simulations are presented to verify the effectiveness and robustness of the proposed control scheme.
\end{abstract}

\section{Key Word:}

fractional-order chaotic system; chaos synchronization; uncertain parameters

\section{Academic Discipline And Sub-Disciplines :}

Applied Mathematics/ Control Systems and Analysis

\section{Mathematics Subject Classification:}

O 415.5

\section{The project name:}

Tianjin Natural Science Foundation: 15JCQNJC01600,

The National Science Foundation of China: 11302148, 11501414.

\section{Council for Innovative Research}

Peer Review Research Publishing System

Journal: JOURNAL OF ADVANCES IN MATHEMATICS

Vol .11, No.6

www.cirjam.com, editorjam@gmail.com 


\section{INTRODUCTION}

Fractional calculus is a 300-year-old topic. Although it has a long mathematical history, the applications of fractional calculus to physics and engineering are just a recent focus of interest. The concept of fractional calculus has tremendous potential to change the way we see, model, and control the nature around us. Compared with the classical integer order models, fractional order derivatives provide an excellent instrument for the description of memory and hereditary properties of various materials and processes.

Synchronization of chaos is a phenomenon that can occur when two or more chaotic systems are coupled or one chaotic system drives the other. In the last few decades, chaos synchronization have received lots of attention from the scientists and researchers, due to its potential applications in chemical reactions, biological systems, information processing, secure communications, etc ${ }^{[1-5]}$. The pioneering work of Pecore and Carrol ${ }^{[6]}$ introduce a method about synchronization between identical and non identical systems with different initial conditional. And to date many methods and techniques have been developed, such as complete synchronization, anti-synchronization, phase synchronization, lag synchronization, intermittent lag synchronization, generalized synchronization, intermittent generalized synchronization, time scale synchronization, projective synchronization, and so on ${ }^{[7-15]}$.

In 2002, S. Chen ${ }^{[16]}$ presented a novel parameter identification and synchronization method for non-identical chaotic systems with unknown parameters based on adaptive control method. In 2006, H.Zhang ${ }^{[17]}$ proposed a method for adaptive synchronization of two chaotic systems with different structure and unknown parameters. In 2007, Yan and $\mathrm{Li}^{[18]}$ presented chaos synchronization of fractional order Lorenz, Rossler and Chen systems taking one as master and second one as slave. In 2008, Zhou and Cheng ${ }^{[19]}$ synchronized between different fractional order chaotic systems.

In this paper the adaptive synchronization of a class of fractional order chaotic system with uncertain parameters is investigated. A reasonable controller and parameter identification rules are designed and proved by $\mathrm{J}$ function criterion. At last, numerical simulation coincide with the theoretical analysis.

\section{Preliminaries, problem description}

\subsection{Fractional calculus}

Fractional calculus is a generalization of integration and differentiation to a non-integer order integro-differential operator

$$
\begin{aligned}
& { }_{a} D_{t}^{q} \text {, which is defined by } \\
& { }_{a} D_{t}^{q}=\left\{\begin{array}{cc}
\frac{d^{q}}{d t^{q}}, & R(q)>0, \\
1, & R(q)=0, \\
\int_{a}^{t}(d \tau)^{-q}, & R(q)<0,
\end{array}\right.
\end{aligned}
$$

Where $q$ is the fractional order which can be a complex number, $R(q)$ denotes the real part of $q$ and $a<t, a$ is the fixed lower terminal \& $t$ is the moving upper terminal.

There are some definitions for fractional derivative.

Definition 1. A real function $f(t), t>0$, is said to be in the space $C_{\mu}, \mu \in \mathfrak{R}$, if there exists a real number $p>\mu$, such that $f(t)=t^{p} f_{1}(t)$, where $f_{1}(t) \in C(0, \infty)$, and it is said to be in the space $C_{\mu}^{n}$ if and only if $f^{(n)} \in C_{\mu}, n \in N$. 
Definition 2. The Riemann-Liouville fractional integral operator ( $J_{t}^{\alpha}$ )of order $\alpha>0$, of a function $f \in C_{\mu}$, $\mu \geq-1$, is defined as

$J_{t}^{\alpha} f(t)=\frac{1}{\Gamma(\alpha)} \int_{0}^{t}(t-\xi){ }^{\alpha-1} f(\xi) d \xi$

where $\alpha>0, t>0, \Gamma($.$) is the well-known gamma function.$

Definition 3. The fractional derivative $D_{t}^{\alpha}$ of $f(t)$, in the Caputo sense is defined as

$D_{t}^{\alpha} f(t)=\frac{1}{\Gamma(n-\alpha)} \int_{0}^{t}(t-\xi)^{n-\alpha-1} f^{(n)}(\xi) d \xi$

for $n-1<\alpha<n . n \in N, t>0, f \in C_{-1}^{n}$.

The important reason of choosing Caputo derivatives for solving initial value fractional order differential equation is that the Caputo derivative of a constant is zero, whereas the Riemann-Liouville fractional derivative of constant is not equal to zero.

\subsection{Numerical approximation method}

Numerical methods used for solving ODEs have to be modified for solving fractional differential equations (FDEs). We only derive the predictor-corrector scheme for drive-response systems. This scheme is the generalization of Adams-Bashforth-Moulton one. We interpret the approximate solution of non-linear fractional-order differential equations by means of this algorithm in the following way.

The considered differential equation,

$D_{t}^{q} y(t)=f(t, y(t)), \quad 0 \leq t \leq T, \quad y^{(k)}(0)=y_{0}^{(k)}, \quad k=0,1, \ldots,[q]$,

is equivalent to the Volterra integral equation

$y(t)=\sum_{k=0}^{[q]-1} y_{0}^{(k)} \frac{t^{k}}{k !}+\frac{1}{\Gamma(q)} \int_{0}^{t}(t-s)^{q-1} f(s, y(s)) d s$.

Set $h=T / N, \quad t_{n}=n h, \quad n=0,1, \ldots, N \in Z^{+}$.

Then $y(t)$ can be described as follows:

$y_{h}\left(t_{n+1}\right)=\sum_{k=0}^{[q]-1} y_{0}^{(k)} \frac{t_{n+1}^{k}}{k !}+\frac{h^{q}}{\Gamma(q+2)} f\left(t_{n+1}, y_{h}^{p}\left(t_{n+1}\right)\right)+\frac{h^{q}}{\Gamma(q+2)} \sum_{j=0}^{n} a_{j, n+1} f\left(t_{h}, y_{h}\left(t_{j}\right)\right)$,

$q_{j, n+1}=\left\{\begin{array}{cc}n^{q+1}-(n-q)(n+1)^{q} & \text { if } j=0 \\ (n-j+2)^{q+1}+(n-j)^{q+1}-2(n-j+1)^{q+1} & \text { if } 0 \leq j \leq n \\ 1 & \text { if } j=n+1\end{array}\right.$

where the predicted value $y_{h}\left(t_{n+1}\right)$ is determined by 


$$
\begin{aligned}
& y_{h}^{p}\left(t_{n+1}\right)=\sum_{k=0}^{[q]-1} y_{0}^{(k)} \frac{t_{n+1}^{k}}{k !}+\frac{1}{\Gamma(q)} \sum_{j=0}^{n} b_{j, n+1} f\left(t_{j}, y_{h}\left(t_{j}\right)\right), \\
& b_{j, n+1}=\frac{h^{q}}{q}\left((n+1-j)^{q}-(n-j)^{q}\right) .
\end{aligned}
$$

The error estimate is

$$
\max _{j=0,1, \ldots, N}\left|y\left(t_{j}\right)-y_{h}\left(t_{j}\right)\right|=o\left(h^{p}\right)
$$

where

$$
p=\min (2,1+q)
$$

\section{3 problem description}

Consider a master system in the form of

$$
\frac{d^{q} x}{d t^{q}}=f(t, x)
$$

and the slave system in the form of

$\frac{d^{q} y}{d t^{q}}=g(t, y)+u(t, x, y)$

where $x=\left(x_{1}, x_{2} \ldots x_{n}\right)^{T} \in R^{n}, y=\left(y_{1}, y_{2} \ldots y_{n}\right)^{T} \in R^{n}$ are the state vectors, $0<q<1, u(t, x, y)$ is a suitable controller.

Let $e=y-x$ is synchronization error vector. The synchronization can be accomplished if it satisfies $\lim _{t \rightarrow \infty}\|e\|=\lim _{t \rightarrow \infty}\left\|y\left(t, y_{0}\right)-x\left(t, x_{0}\right)\right\|=0$, where $\|$.$\| is the Euclidean norm.$

Theorem 1. Stability theorem of fractional-order system

Consider the following nonlinear system of fractional differential equation.

$$
\frac{d^{q} x}{d t^{q}}=A(x) x
$$

System (5) is locally asymptotically stable if all the eigenvalues $\left(\lambda_{1}, \ldots, \lambda_{n}\right)$ of the Jacobian matrix of all equilibrium point satisfy

$$
\left|\arg \left(\lambda_{i}\right)\right|>\frac{\pi}{2} q
$$

The stable and unstable regions for $q \in(0,1]$ are depicted in Fig 1 . 


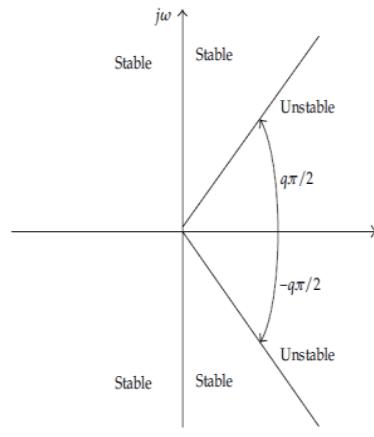

Fig 1: Stability region of fractional-order system

\section{System description}

The system considered in this paper is the following

$$
\left\{\begin{array}{l}
\frac{d^{q} x}{d t^{q}}=a(y-x) \\
\frac{d^{q} y}{d t^{q}}=b x+c y-x z \\
\frac{d^{q} z}{d t^{q}}=x^{2}-d z
\end{array}\right.
$$

(7)

where $x, y, z$ are the state variables, and the parameters' values are $a=20, b=14, c=10.6, d=2.8$. This system has three equilibria:

$S_{1}=(0,0,0)$

$S_{2}=(8.2994,8.2994,24.6)$

(8)

$S_{3}=(-8.2994,-8.2994,24.6)$

The corresponding Jacobian matrix is as follows:

$J=\left(\begin{array}{ccc}-a & a & 0 \\ b-z & c & -x \\ 2 x & 0 & -d\end{array}\right)$

Then , the eigenvalues of the Jacobian matrix are obtained:

$S_{1}: \lambda_{1}=-2.8, \lambda_{2}=17.9736, \lambda_{3}=-27.3736$,

$S_{2}: \lambda_{1}=-18.6839, \lambda_{2}=3.2419+11.7027 i, \lambda_{3}=3.2419-11.7027 i$,

$S_{3}: \lambda_{1}=-18.6839, \lambda_{2}=3.2419+11.7027 i, \lambda_{3}=3.2419-11.7027 i$,

According to theorem 1, we know $\left|\arg \left(\lambda_{1}\right)\right|=\left|\arg \left(\lambda_{2}\right)\right| \approx \frac{0.8284}{2} \pi$, so the system is chaotic when $0.8284<q<1$.

The chaotic attractors of the system for the order of derivative $q=0.9$ and $q=0.99$ are displayed through Fig.2 and Fig.3. 


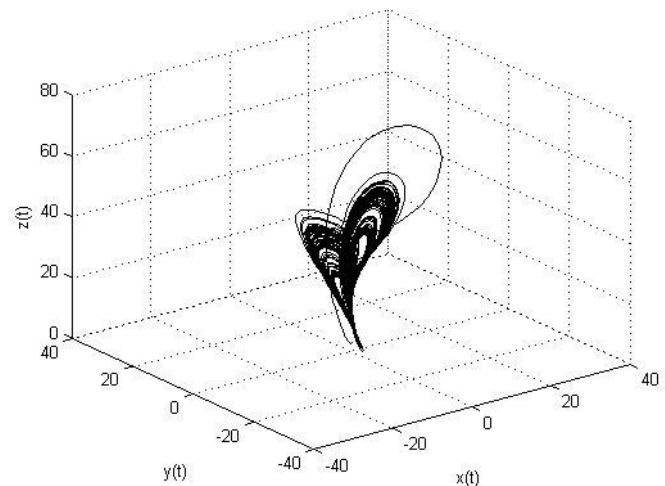

Fig.2 Phase plot of the system with $q=0.9$

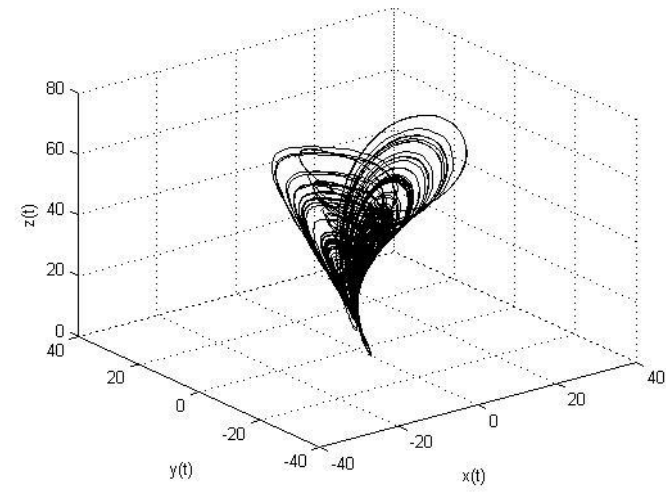

Fig.3 Phase plot of the system with $q=0.99$

\section{Adaptive synchronization and parameter identification}

In this section, adaptive synchronization of the fractional order chaotic system with uncertain parameters will be studied. Theorem $2^{[20]}$. J function criterion.

For one fractional order system, $\frac{d^{q} x}{d t^{q}}=A x, 0<q \leq 1$, if there exists a real symmetric positive definite matrix $P$, such that $J=x^{T} P \frac{d^{q} x}{d t^{q}} \leq 0$ for every state variables $x$. Then the fractional order system asymptotically stable.

For this, we define the drive system (11) and the response system (12) with control parameters as

$$
\left\{\begin{array}{l}
\frac{d^{q} x_{1}}{d t^{q}}=a\left(x_{2}-x_{1}\right) \\
\frac{d^{q} x_{2}}{d t^{q}}=b x_{1}+c x_{2}-x_{1} x_{3} \\
\frac{d^{q} x_{3}}{d t^{q}}=x_{1}^{2}-d x_{3}
\end{array}\right.
$$

and

$$
\left\{\begin{array}{l}
\frac{d^{q} y_{1}}{d t^{q}}=\bar{a}\left(y_{2}-y_{1}\right)+u_{1} \\
\frac{d^{q} y_{2}}{d t^{q}}=\bar{b} y_{1}+\bar{c} y_{2}-y_{1} y_{3}+u_{2} \\
\frac{d^{q} y_{3}}{d t^{q}}=y_{1}^{2}-\bar{d} y_{3}+u_{3}
\end{array}\right.
$$

where $u_{1}, u_{2}, u_{3}$ are the control parameters.

In order to facilitate the following analysis, the error functions are defined as

$$
e_{1}=y_{1}-x_{1}, e_{2}=y_{2}-x_{2}, e_{3}=y_{3}-x_{3}
$$

and

$e_{a}=\bar{a}-a, e_{b}=\bar{b}-b, e_{c}=\bar{c}-c, e_{d}=\bar{d}-d$ 
Theorem 3. If control functions are selected as

$\left\{\begin{array}{l}u_{1}=-a e_{2} \\ u_{2}=y_{1} y_{3}-x_{1} x_{3}+k e_{2}-b e_{1} \\ u_{3}=-y_{1}^{2}+x_{1}^{2}\end{array}\right.$

where $k \leq-c$ is the real constants, and adaptive laws of parameters are calculated as

$\left\{\begin{array}{l}\frac{d^{q} e_{a}}{d t^{q}}=e_{1} y_{1}-e_{1} y_{2} \\ \frac{d^{q} e_{b}}{d t^{q}}=-e_{2} y_{1} \\ \frac{d^{q} e_{c}}{d t^{q}}=-e_{2} y_{2} \\ \frac{d^{q} e_{d}}{d t^{q}}=e_{3} y_{3}\end{array}\right.$

then, the response system (12) is synchronized with the drive system (11) globally and asymptotically, i.e. $\lim _{t \rightarrow \infty}\|e(t)\|=0$.

Proof: From Eq.(13), (14),(15)and (16), the error systems are obtained as

$\left\{\begin{array}{l}\frac{d^{q} e_{1}}{d t^{q}}=e_{a} y_{2}-e_{a} y_{1}-a e_{1} \\ \frac{d^{q} e_{2}}{d t^{q}}=e_{b} y_{1}+e_{c} y_{2}+c e_{2}+k e_{2} \\ \frac{d^{q} e_{3}}{d t^{q}}=-e_{d} y_{3}-d e_{3}\end{array}\right.$

Let us define a $\mathrm{J}$ function as follows

$$
\begin{aligned}
& J=\left[e_{1}, e_{2}, e_{3}, e_{a}, e_{b}, e_{c}, e_{d}\right] \cdot P_{7 \times 7} \cdot\left[\frac{d^{q} e_{1}}{d t^{q}}, \frac{d^{q} e_{2}}{d t^{q}}, \frac{d^{q} e_{3}}{d t^{q}}, \frac{d^{q} e_{a}}{d t^{q}}, \frac{d^{q} e_{b}}{d t^{q}}, \frac{d^{q} e_{c}}{d t^{q}}, \frac{d^{q} e_{d}}{d t^{q}}\right]^{T} \\
& =e_{1} \frac{d^{q} e_{1}}{d t^{q}}+e_{2} \frac{d^{q} e_{2}}{d t^{q}}+e_{3} \frac{d^{q} e_{3}}{d t^{q}}+e_{a} \frac{d^{q} e_{a}}{d t^{q}}+e_{b} \frac{d^{q} e_{b}}{d t^{q}}+e_{c} \frac{d^{q} e_{c}}{d t^{q}}+e_{d} \frac{d^{q} e_{d}}{d t^{q}} \\
& =e_{1}\left(e_{a} y_{2}-e_{a} y_{1}-a e_{1}\right)+e_{2}\left(e_{b} y_{1}+e_{c} y_{2}+c e_{2}+k e_{2}\right)+e_{3}\left(-e_{d} y_{3}-d e_{3}\right) \\
& +e_{a}\left(e_{1} y_{1}-e_{1} y_{2}\right)+e_{b}\left(-e_{2} y_{1}\right)+e_{c}\left(-e_{2} y_{2}\right)+e_{d}\left(e_{3} y_{3}\right) \\
& =-a e_{1}^{2}+(k+c) e_{2}^{2}-d e_{3}^{2} \leq 0
\end{aligned}
$$

where $k \leq-c$ is the real constants, so according to theorem 2, the response system (12) is synchronization to the drive system (11) asymptotically.

\section{Numerical simulation}

In the numerical simulations, we set the parameters of the system (11) as $a=20, b=14, c=10.6, d=2.8$. The constant is taken as $k=-15$. 
Case 1. when $q=0.9$, the initial conditions of the drive and response systems are taken arbitrarily as $\left(x_{1}(0), x_{2}(0), x_{3}(0)\right)=(1,1,2)$, and $\left(y_{1}(0), y_{2}(0), y_{3}(0)\right)=(-3,3,0)$. The initial values of the estimated unknown parameter vectors of the systems are taken as $(\bar{a}, \bar{b}, \bar{c}, \bar{d})=(15,10,6,0)$. Fig. 4 (a) shows that the error vector $e_{1}$ converges asymptotically to zero. Fig. 4 (b) shows that the error vector $e_{2}$ also converges asymptotically to zero. The curve of synchronization error rector $e_{3}$ is shown in Fig. 4 (c). Fig. 4 (a)-(c) clearly exhibit that the response system will be adaptive synchronized with the drive system, and Fig. 5 shows that the estimated parameter vectors converge to the original parameter vectors.

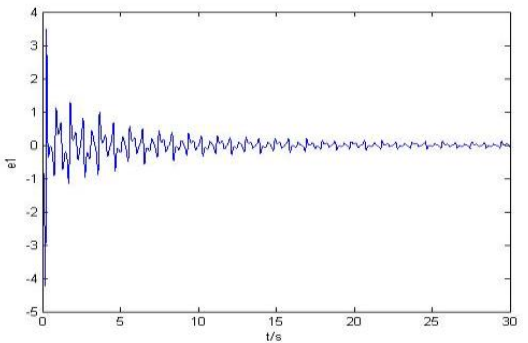

(a)

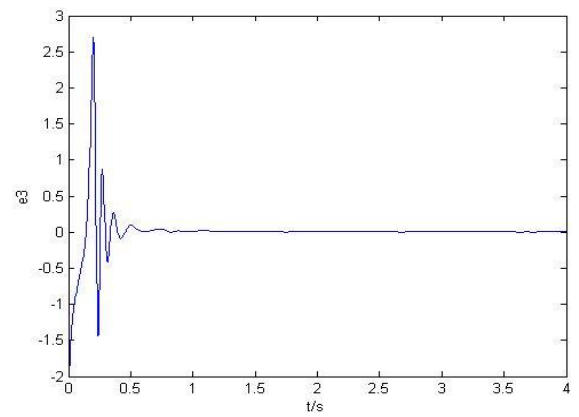

(c)

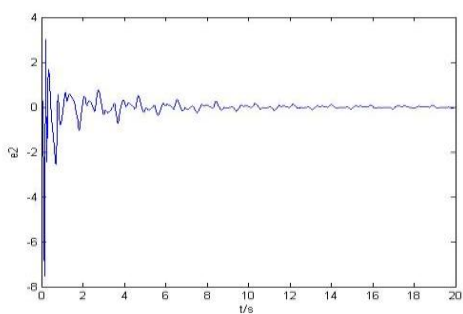

(b)

Fig.4 State trajectories of the errors function of drive system (11) \& response system (12)

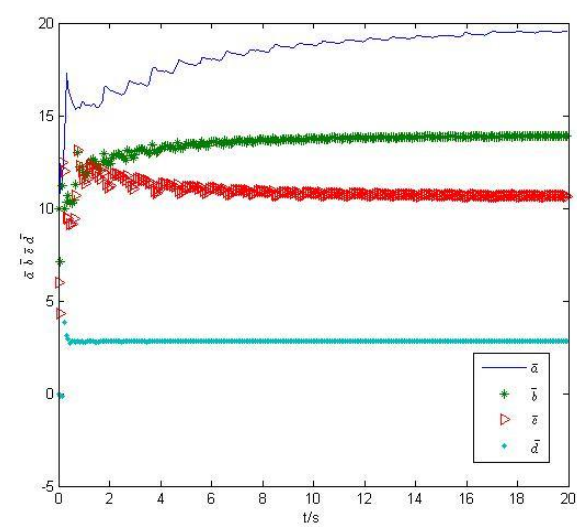

Fig.5 State trajectories of the estimated parameter vectors $\bar{a}, \bar{b}, \bar{c}, \bar{d}$ 
Case 2. when $q=0.99$, the initial conditions of the drive and response systems are taken arbitrarily as $\left(x_{1}(0), x_{2}(0), x_{3}(0)\right)=(2,3,5)$, and $\left(y_{1}(0), y_{2}(0), y_{3}(0)\right)=(-5,8,0)$. The initial values of the estimated unknown parameter vectors of the systems are taken as $(\bar{a}, \bar{b}, \bar{c}, \bar{d})=(0,20,18,-5)$. Fig. 6 (a) shows that the error vector $e_{1}$ converges asymptotically to zero. Fig. 6 (b) shows that the error vector $e_{2}$ also converges asymptotically to zero. The curve of synchronization error rector $e_{3}$ is shown in Fig. 6 (c). Fig. 6 (a)-(c) clearly exhibit that the response system will be adaptive synchronized with the drive system, and figure 7 shows that the estimated parameter vectors converge to the original parameter vectors.

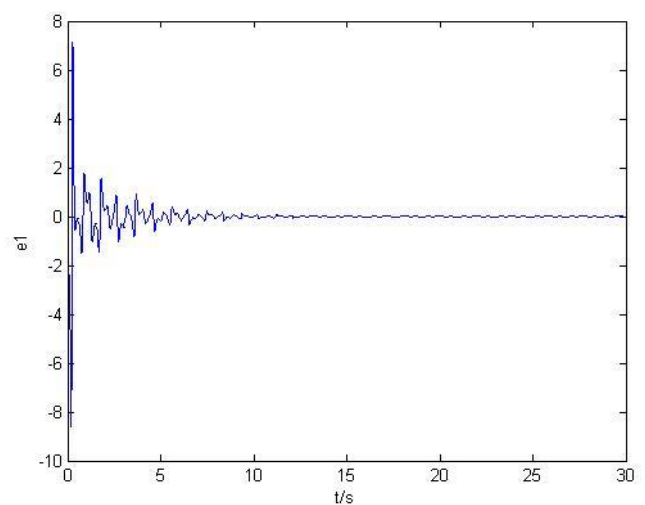

(a)

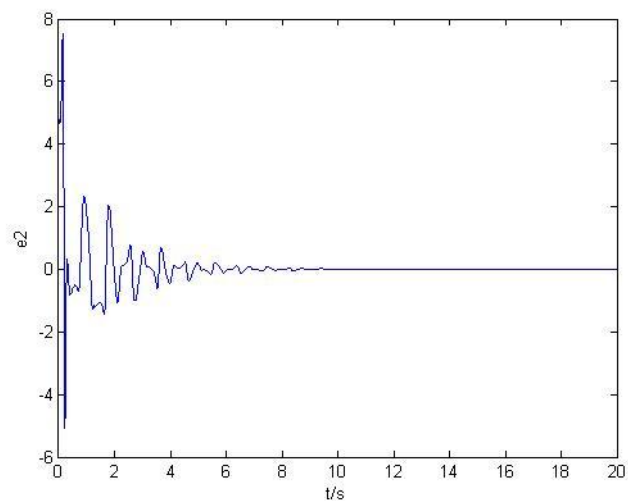

(b)

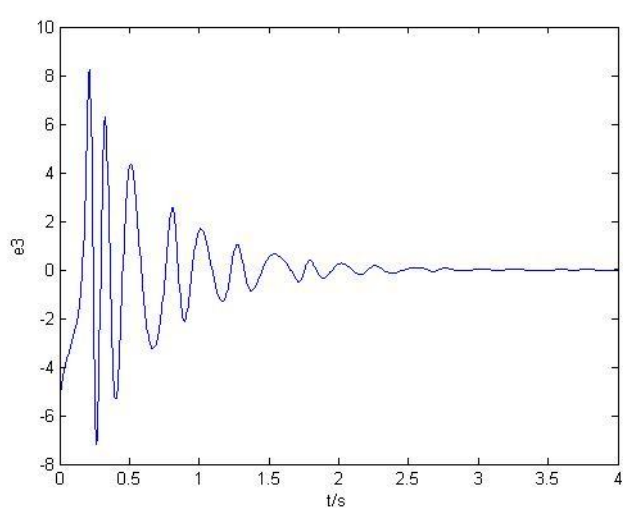

(c)

Fig.6 State trajectories of the errors function of drive system (11) \& response system (12) 


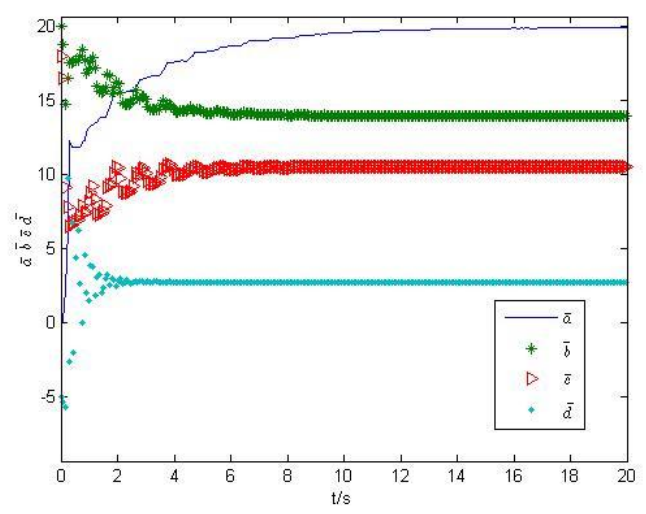

Fig.7 State trajectories of the estimated parameter vectors $\bar{a}, \bar{b}, \bar{c}, \bar{d}$

By comparing Case I and Case II, the results demonstrate that the time of synchronization of $e_{1}, e_{2}$ in Case II is slightly shorter than the Case I , and the time of $e_{3}, \bar{a}, \bar{b}, \bar{c}, \bar{d}$ in Case I is shorter than the Case II.

\section{Conclusion}

In this letter, the adaptive synchronization of a class of fractional order chaotic system with uncertain parameters is presented, and based on the fractional stability criterion, the local stability of the fractional order chaotic system is analyzed. Besides, suitable adaptive synchronization controller and parameter identification rules of the unknown parameters are investigated by using the $\mathrm{J}$ function criterion. Numerical simulations are performed to verify these results.

\section{Reference}

[1] Yang T, Chua LO. 1996. Secure communication via chaotic parameter modulation. IEEE Trans Circuits Syst I. Vol. 43, 817-819.

[2] Feki M. 2003. An adaptive chaos synchronization scheme applied to secure communication. Chaos Soliton Fract. Vol. 3, 959-964.

[3] Li C, Liao X, Wong K. 2004. Chaotic lag synchronization of coupled time-delayed systems and its application in secure communication. Physica D. Vol. 194, 187-202.

[4] Chang WD. 2009. Digital secure comumunication via chaotic systems. Digital Signal Process . Vol. 19, $693-699$.

[5] Nana B, Woafo P, Domngang S. 2009. Chaotic synchronization with experimental application to secure communication. Commun Nonlinear Sci Numer Simul. VOI. 14, 2266-2276.

[6] L. M. Pecora, T. L. Carrol. 1990. Synchronization in chaotic system. Phys. Rev. Lett. Vol. 64, 821-824.

[7] Carrol TL, Heagy Jf, Pecora LM. 1996. Transforming signals with chaotic synchronization. Phys Rev E. Vol. 54, 4676-4680.

[8] Zhang Y, Sun J. 2004. Chaotic synchronization and anti-synchronization based on suitable separation. Phys Lett A . Vol. 330, 442-447.

[9] Rosenblum MG. Pikovsky AS, Kurths J. 1996. Phase synchronization of chaotic oscillators. Phys Rev Lett. Vol. 76, 1804-1807.

[10] Rosenblum MG. Pikovsky AS, Kurths J. 1997. From phase to lag synchronization in coupled chaotic oscillators. Phys Rev Lett. Vol. 78, 4193-4196.

[11] Boccaletti S. Valladares DL. 2000. Characterization of intermittent lag synchronization. Phys Rev E. Vol. 62, 7497-7500.

[12] Rulkov NF, Sushchik MM, Tsimring LS. 1995. Generalized synchronization of chaos in directionally coupled chaotic systems. Phys Rev E. Vol. 51, 980-994. 
[13] Hramov AE, Koronovskii AA, Moskalenko OI. 2005. Generalized synchronization onset. Europhys Lett. Vol. 72 , 901-910.

[14] Hramov AE, Koronovskii AA. 2004. An approach to chaotic synchronization. Chaos, Vol. 14, 603-610.

[15] Mainieri R, Rehacek J. 1999. Projective synchronization in three-dimensional chaotic system. Phys Rev Lett. Vol. 82 , 3042-3045.

[16] Chen SH, Lu J. 2002. Parameters identification and synchronization of chaotic systems based upon adaptive control. Phys. Lett. A. Vol. 299, 353-358.

[17] Zhang H, Huang W, Wang Z, Chai T. 2006. Adaptive synchronization between two different chaotic systems with unknown parameters. Phys. Lett. A. Vol. 350, 363-366.

[18] Yan JP, Li CP. 2007. On chaos synchronization of fractional differential equations. Chaos Soliton Fract. Vol. 32, 725-735.

[19] Zhou P, Cheng X. 2008. Synchronization between different fractional order chaotic systems. In: Proceeding of the 7th world congress on intelligent control and automation, Chongqing, China.

[20] Hu J B, Han Y, Zhao L D. 2009. Anovel stability theorem fractional systems and its applying in synchronizing fractional chaotic system based on back-stepping approach. Acta Physica Sinica. Vol. 58, 2235-2239. 